\title{
Invention of an Original Tetra-Generations Patch Antenna for the New Generation of Mobile Telephony and the Study of the Thermal Effect of GSM on the Human Head and Hand
}

\author{
Jalal Baayer ${ }^{1}$ and Hassan Ammor ${ }^{2}$ \\ \{jalal.baayer@gmail.com ${ }^{1}$, ammor@emi.ac.ma² \\ Smart Communications Research Team, E3S Research Center, Engineering Mohammadia School, \\ Mohammed V University, Rabat, Morocco ${ }^{1}$ \\ Smart Communications Research Team, E3S Research Center, Engineering Mohammadia School, \\ Mohammed V University, Rabat, Morocco ${ }^{2}$
}

\begin{abstract}
The present invention relates to a miniature original tetra-generations patch antenna with rectangular slots at low cost, and dedicated to the new generation of mobile telephony by covering the frequency bands of $900 \mathrm{MHz}$, $1800 \mathrm{MHz}, 2100 \mathrm{MHz}$ and $2600 \mathrm{MHz}$. The Modeling and simulation of the basic model are performed using the Ansoft HFSS simulator employing finite element method (FEM). This study allowed us to determine the reflection coefficient, the voltage standing wave ratio (VSWR), the omnidirectional radiation and the bandwidth. With this antenna, we obtained new results from the GSM thermal effect on the human head and hand. With this in mind, a new, simple and miniaturized quadri-band antenna in patch technology meeting the standards of dosimetry and temperature for mobile phone applications is designed and proposed.
\end{abstract}

Keywords: Tetra-generations patch antenna, SAR, human head, human hand, dosimetry, temperature rise.

\section{Introduction}

Our study constitutes the simulation and the realization of a new tetra-generations patch antenna for the new generations of the mobile telephony operating in the bands of frequencies of $900 \mathrm{MHz}, 1800 \mathrm{MHz}, 2100 \mathrm{MHz}$ and $2600 \mathrm{MHz}$ respecting the norms of the dosimetry and the temperature.

This work is based on simulations obtained on the Ansoft HFSS software basing on two anatomical models. One concerning the human head and composed of seven layers and the other relating to the human hand and represented by 4 layers. The simulations of the SAR distribution and temperature rise are performed at four frequency bands, for seven antennahead distances for the head and one distance for the hand during five exposure times, based on the tetra-generations patch antenna model. 


\section{Structure of the invented antenna}

\subsection{Basic structure adopted for the design}

The geometry of the invented antenna is represented in figure 1 , the antenna is made on a FR4_epoxy substrate of dimensions $\mathrm{L} \times \mathrm{W}=45 \times 42 \mathrm{~mm}^{2}$ with a dielectric constant $\mathrm{\varepsilon r}=4.4$ [1]. The thickness of the substrate is $h=1.6 \mathrm{~mm}$. The antenna consists of a square patch having optimal characteristics and reduced dimensions and including technical slots.

The value of the permittivity $\varepsilon r$ is chosen such that it provides a better efficiency and effectiveness and a wider bandwidth. The antenna power is supplied via a $50 \Omega$ coaxial power supply to improve bandwidth and gain.

We performed the simulation with the HFSS software (High Frequency Structure Simulator). We chose the substrate, calculated the width, the length, the effective dielectric constant, which allowed us to have the structure proposed in figure 1.

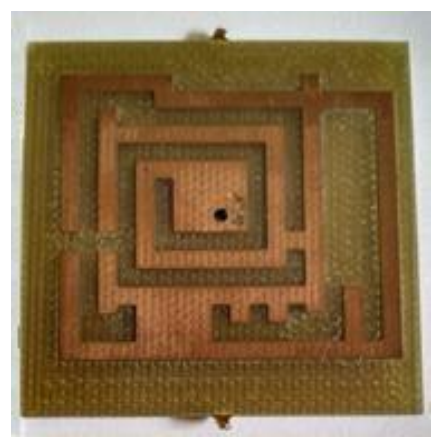

Fig. 1. The invented and performed tetra-generations patch antenna.

The figure 2 shows the reflection coefficient of the patch antenna presented from the simulation based on the Ansoft HFSS software.

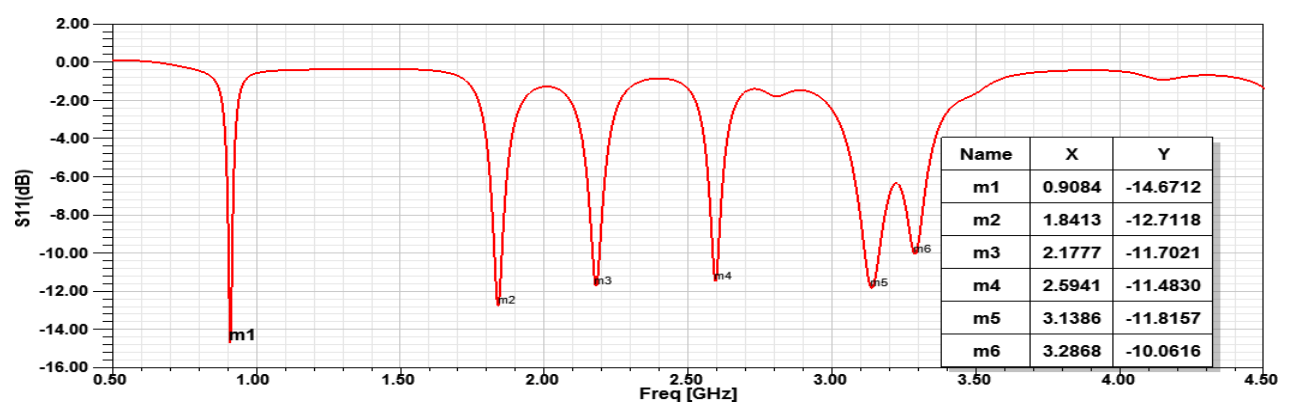

Fig. 2. The reflection coefficient of the invented antenna. 
The figure 3 illustrates the comparison between the real results and the simulation of the reflection coefficient $\mathrm{S} 11$ of the tetra-generations patch antenna.

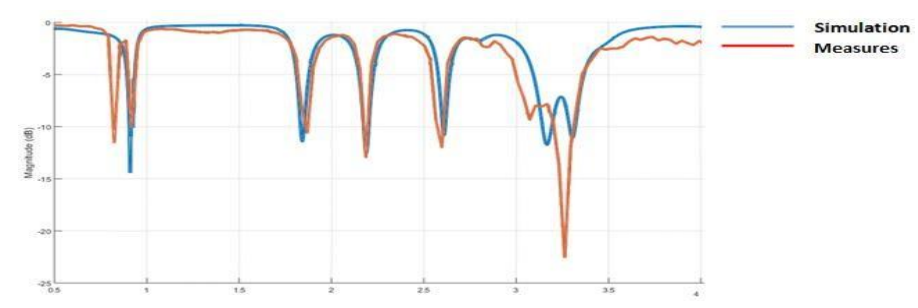

Fig. 3. Measures and simulation results of the tetra-generations patch antenna reflection coefficient.

\section{SAR distribution and temperature rise}

The SAR is represented by the following formula [2]:

$$
S A R=\frac{\sigma}{2 \rho}|E|^{2} \quad(1)
$$

With $\sigma$ the electrical conductivity $(\mathrm{S} / \mathrm{m}), \rho$ is the density of tissue $(\mathrm{kg} / \mathrm{m} 3)$ and $\mathrm{E}$ is the peak value of the electric field $(\mathrm{V} / \mathrm{m})$. The absorption of electromagnetic waves causes a temperature variation $\Delta \mathrm{T}$, in the case of a human body with specific heat $\mathrm{C}$, moreover in thermal equilibrium with the environment, and exposed during the time $\Delta$ t to these waves. [3]:

This correlation between the SAR and the temperature variation is given by the formula

$$
S A R=C \frac{\Delta T}{\Delta t}(2)
$$

According to our recent work, the maximum SAR is observed at the level of the skin of the human head and can be represented according to the formula:

$$
S A R_{\max }=f\left(\theta_{x}, \theta_{y}\right)
$$


And for a fixed $\Delta \mathrm{Z}$ among $\{5,10,15,20,25,30\}(\mathrm{mm})$.

\section{With:}

$\theta \mathrm{x}$ : rotation angle around the $\mathrm{OX}$ axis.

$\theta y$ : rotation angle around the $\mathrm{OY}$ axis.

$\Delta \mathrm{Z}$ : displacement along the $\mathrm{OZ}$ axis.

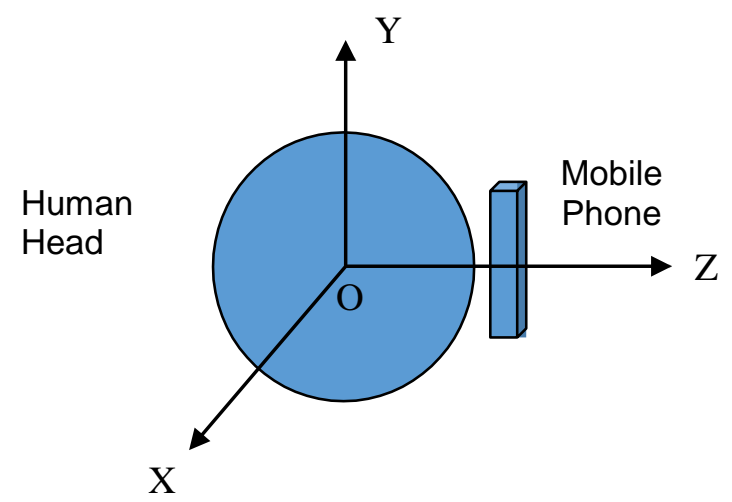

Fig. 4. Positions of the mobile phone and the human head.

\section{Modeling of the human head and hand}

We used in figure 5 a model of flat multilayer human head with seven types of tissues that are: skin, fat, muscle, skull, DM (dura), CSF and brain. Each layer has its intrinsic dielectric properties and its thermal characteristics.

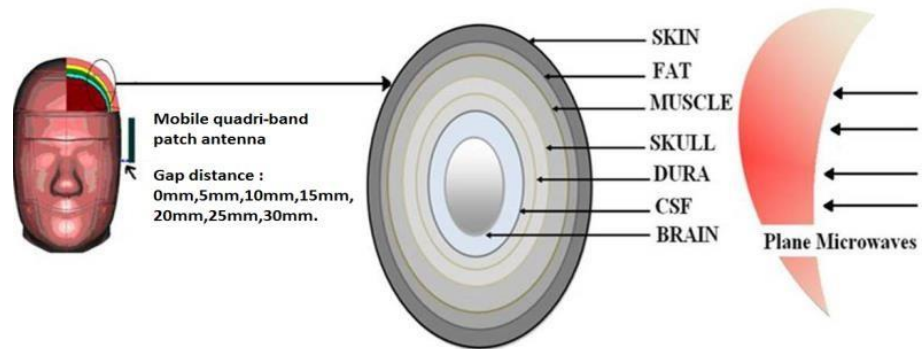

(a)

(b)

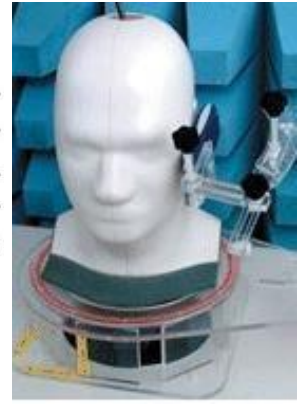

( c)

Fig. 5. The model of the human head with 7 layers and the SAM phantom.

(a) Human head model in cross section with mobile phone.

(b) Irradiation of the human head model consisting of seven layers per electromagnetic waves.

(c) SAM phantom of human head used in a SAR test bench. 
We used according to figure 6 an HFSS model of human hand multilayer with four types of tissues that are: skin, fat, muscle and bone and irradiated by our tetra-generations patch antenna.

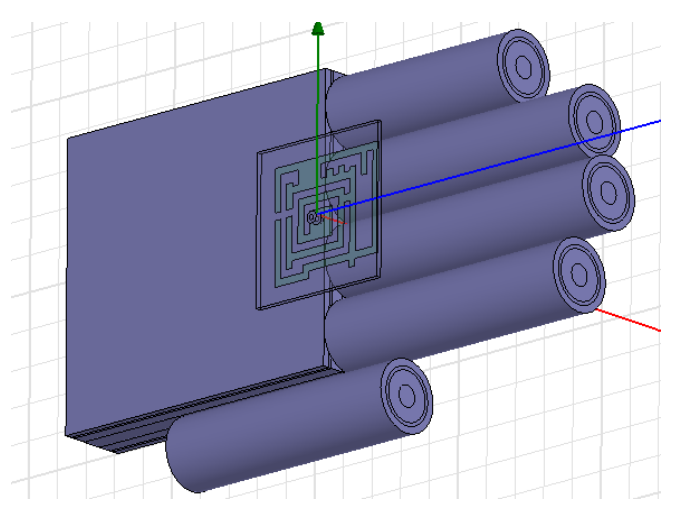

Fig. 6. HFSS model of the human hand irradiated by our tetra-generations patch antenna.

The tables 1 and 2 show the numerical values of the dielectrics, the thermal properties and the mass density of each layer. These values were used from the data of the scientific literature [4]:

Table 1. Dielectric properties of human head and hand models layers.

\begin{tabular}{|c|c|c|c|c|c|c|c|c|}
\hline \multirow[t]{2}{*}{ Layers } & \multicolumn{2}{|c|}{$900 \mathrm{MHz}$} & \multicolumn{2}{|c|}{$1800 \mathrm{MHz}$} & \multicolumn{2}{|c|}{$2100 \mathrm{MHz}$} & \multicolumn{2}{|c|}{$2600 \mathrm{MHz}$} \\
\hline & 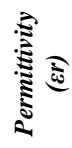 & 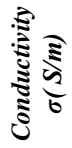 & 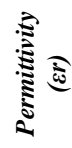 & 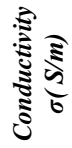 & 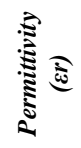 & 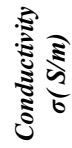 & 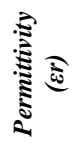 & 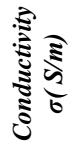 \\
\hline Skin & 43.8 & 0.86 & 43.9 & 1.23 & 43.9 & 1.23 & 42.9 & 1.59 \\
\hline Fat & 11.3 & 0.11 & 11.0 & 0.19 & 11.0 & 0.19 & 10.82 & 0.26 \\
\hline Muscle & 55.9 & 0.97 & 54.4 & 1.38 & 54.3 & 1.38 & 53.64 & 1.77 \\
\hline Bone & 20.8 & 0.34 & 15.6 & 0.43 & 15.5 & 0.43 & 15.01 & 0.57 \\
\hline Dura & 44.4 & 0.96 & 42.9 & 1.32 & 42.9 & 1.32 & 42.03 & 1.66 \\
\hline CSF & 68.6 & 2.41 & 67.2 & 2.92 & 67.2 & 2.92 & 66.24 & 3.45 \\
\hline Brain & 45.8 & 0.77 & 43.5 & 1.15 & 43.4 & 1.15 & 42.61 & 1.48 \\
\hline
\end{tabular}


Table 2. Thermal properties and mass density of the layers of the human head and hand model.

\begin{tabular}{|l|c|c|}
\hline Layers & $\begin{array}{c}\text { Mass Density } \\
\boldsymbol{\rho}(\mathbf{k g} / \mathbf{m 3})\end{array}$ & $\begin{array}{c}\text { Specific heat } \\
(\mathbf{J} / \mathbf{g} . \mathbf{C})\end{array}$ \\
\hline Skin & 1100 & 3.6 \\
\hline Fat & 1100 & 3.0 \\
\hline Muscle & 1040 & 3.5 \\
\hline Bone & 1850 & 3.1 \\
\hline Dura & 1030 & 3.6 \\
\hline CSF & 1030 & 4.0 \\
\hline Brain & 1030 & 3.7 \\
\hline
\end{tabular}

\section{Results and discussions}

\subsection{Mobile thermal effect on the human head}
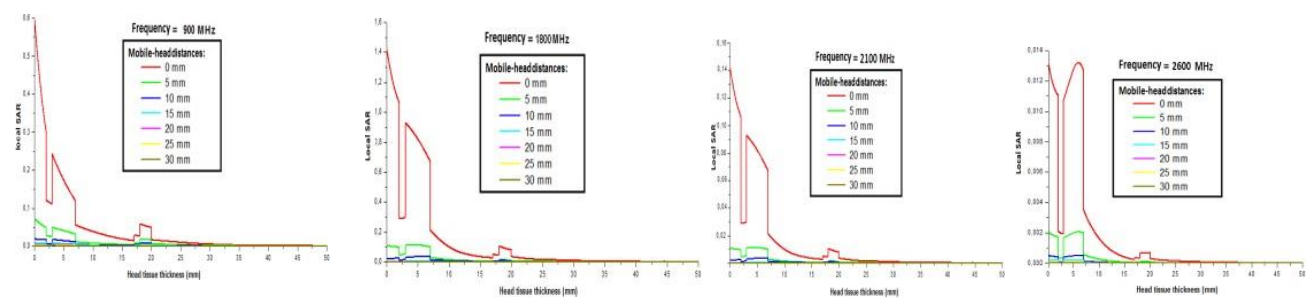

Fig. 7. Distribution of local SAR in the human head.
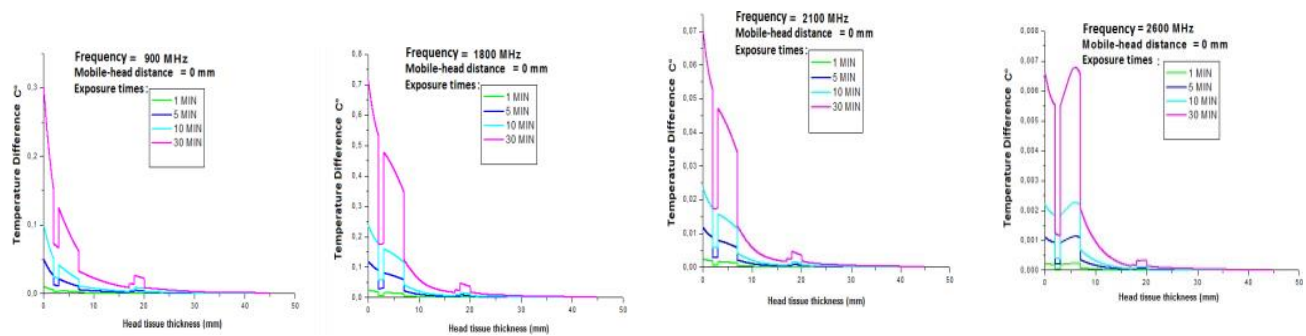

Fig. 8. Spatial distribution of temperature in the human head.

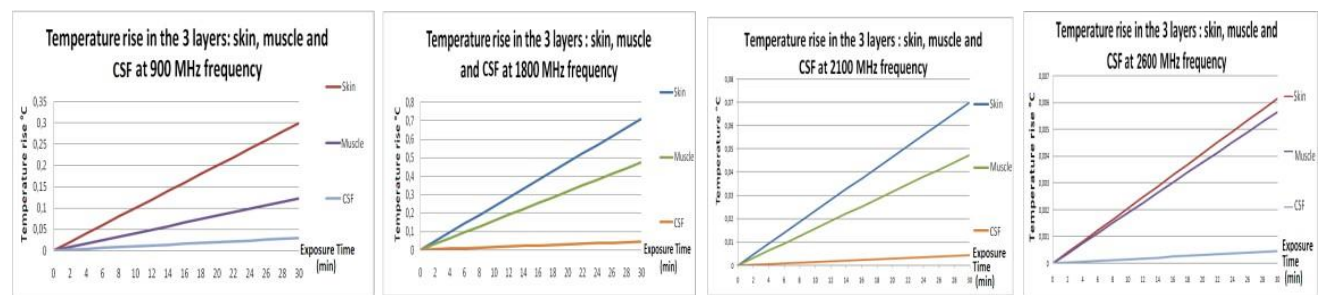

Fig. 9. The increase in temperature in the three layers: skin, muscle and CSF of the human head. 
The figure 7 represents the distribution of local SAR in the human head. The figure 8 shows the spatial distribution of temperature in the human head. In the figure 9 we find the increase in temperature in the three layers: skin, muscle and CSF of the human head.

In the figure 7 , we notice that:

- For each mobile-head distance, the SAR has 3 peaks in the three layers : skin, muscle and CSF.

- As we move away from the skin to the brain, the value of SAR decreases and has very low value in the brain.

- $\quad$ The SAR value decreases as the frequency rises from $900 \mathrm{Mhz}$ and $1800 \mathrm{Mhz}$ to 2100Mhz and 2600Mhz.

In the figure 8 and 9 , we notice that:

- $\quad$ The temperature has 3 peaks in the three layers : skin, muscle and CSF.

- As we move away from the skin to the brain, the value of temperature decreases and has very low value in the brain.

- The temperature value decreases as the frequency rises from $900 \mathrm{Mhz}$ and 1800Mhz to 2100Mhz and 2600Mhz.

- The temperature value increases as the duration of telephone conversation rises.

We conclude that:

- The 3 layers skin, muscle and CSF have significant water levels so a large absorption of mobile electromagnetic waves by their biological tissues. This explains why we have 3 peaks in SAR and temperature in these 3 layers.

- The 3 layers represent a natural protection for the brain against electromagnetic waves of mobile that is why we have minimum values of SAR and temperature in the human brain.

- The migration of telecom operators from older mobile telephony generations of G1 (900Mhz) and G2 (1800Mhz) to G3 (2100Mhz) and G4 (2600 Mhz) is very beneficial for mobile phone users and this explains why we have minimum SAR and temperature values for the $2100 \mathrm{Mhz}$ and $2600 \mathrm{Mhz}$ frequencies.

- The warmth we feel in the superficial layer of the head during a long telephone conversation is explained by the high SAR and temperature value in the skin of the human head. 
5.2 Mobile thermal effect on the human hand

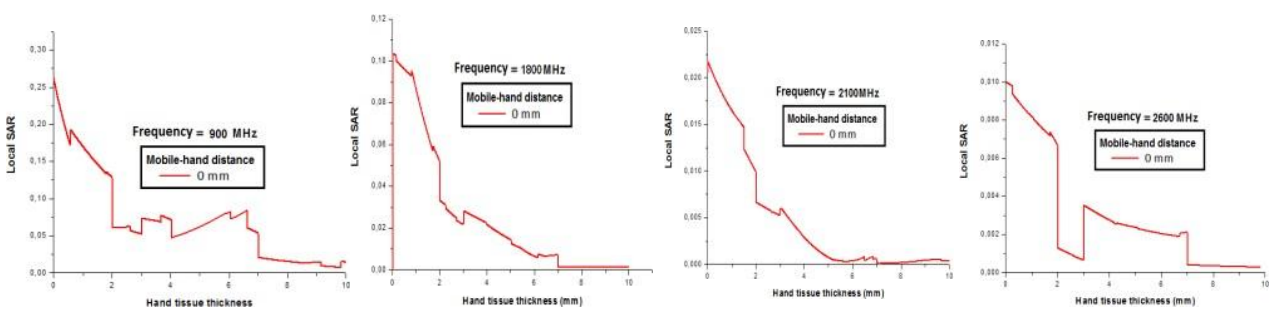

Fig. 10. Distribution of local SAR in the human hand
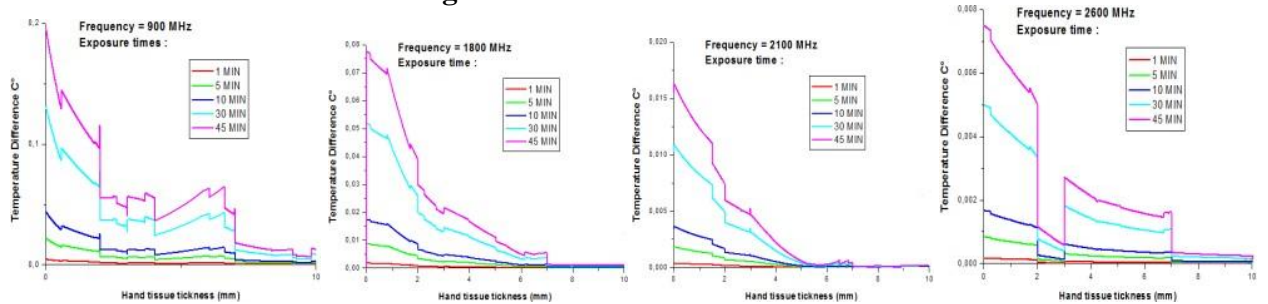

Fig. 11. Spatial distribution of temperature in the human hand.

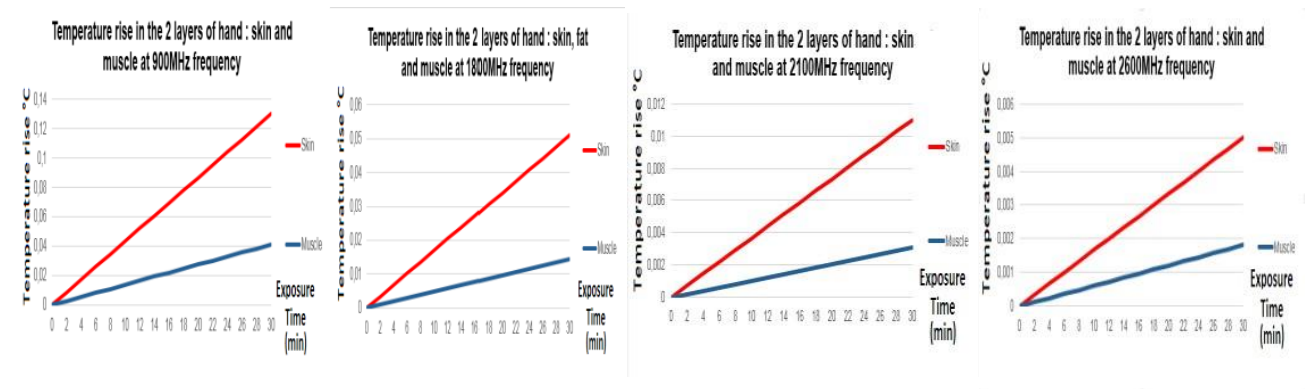

Fig. 12. The increase in temperature in both layers: skin and muscle of the human hand. 
The figure 10 represents the distribution of local SAR in the human hand. The figure 11 shows the spatial distribution of temperature in the human hand. In the figure 12 we find the increase in temperature in the two layers skin and muscle of the human hand.

In the figure 10, we notice that:

- $\quad$ The SAR has 2 peaks in the two layers : skin and muscle.

- As we move away from the skin to the bone, the value of SAR decreases and has very low value in the bone.

- The SAR value decreases as the frequency rises from $900 \mathrm{Mhz}$ and $1800 \mathrm{Mhz}$ to 2100Mhz and 2600Mhz.

In the figure 11 and 12 , we notice that:

- The temperature has 2 peaks in the two layers: skin and muscle.

- As we move away from the skin to the bone, the value of temperature decreases and has very low value in the bone.

- The temperature value decreases as the frequency rises from $900 \mathrm{Mhz}$ and 1800Mhz to 2100Mhz and 2600Mhz.

- The temperature value increases as the duration of telephone conversation rises.

We conclude that:

- The 2 layers skin and muscle have significant water levels so a large absorption of mobile electromagnetic waves by their biological tissues. This explains why we have 2 peaks in SAR and temperature in these 2 layers.

- The 2 layers represent a natural protection for the bone against electromagnetic waves of mobile that is why we have minimum values of SAR and temperature in the bone of human hand.

- The migration of telecom operators from older mobile telephony generations of G1 (900Mhz) and G2 (1800Mhz) to G3 (2100Mhz) and G4 (2600 Mhz) is very beneficial for mobile phone users and this explains why we have minimum SAR and temperature values for the $2100 \mathrm{Mhz}$ and $2600 \mathrm{Mhz}$ frequencies.

- The warmth we feel in the superficial layer of the hand during a long telephone conversation is explained by the high SAR and temperature value in the skin of the human hand. 


\subsection{Mobile and human head inclinations impact on the SAR values}

The table 3 represents SAR values with different inclinations $\theta x \max$ and $\theta y$ max between the human head and the mobile phone for a distance $\Delta \mathrm{Z}=10 \mathrm{~mm}$.

Table 3. SAR values with different inclinations $\theta_{x \max }$ and $\theta_{\mathrm{ymax}}$ between the human head and the mobile phone for a distance $\Delta \mathrm{Z}=10 \mathrm{~mm}$.

\begin{tabular}{|l|l|l|l|l|l|l|}
\cline { 5 - 7 } \multicolumn{2}{l}{} & \multicolumn{5}{c|}{ SAR $_{\max }$} \\
\hline Positions & $\boldsymbol{\theta}_{\text {xmax }}$ & $\boldsymbol{\theta}_{\text {ymax }}$ & $\mathbf{9 0 0 M H z}$ & $\mathbf{1 8 0 0 M H z}$ & $\mathbf{2 1 0 0 M H z}$ & $\mathbf{2 6 0 0 M H z}$ \\
\hline P1 & -30 & 0 & 0,01327 & 0,04407 & 0,00495 & 0,00157 \\
\hline P2 & -20 & 0 & 0,00945 & 0,01973 & 0,00314 & 0,00107 \\
\hline P3 & -10 & 0 & $\mathbf{0 , 0 0 8 1 4}$ & 0,01926 & 0,00190 & 0,00057 \\
\hline P4 & 0 & 0 & 0,00916 & 0,03257 & 0,00193 & 0,00040 \\
\hline P5 & 10 & 0 & 0,01230 & 0,04487 & $\mathbf{0 , 0 0 1 1 9}$ & 0,00084 \\
\hline P6 & 20 & 0 & 0,01480 & 0,04561 & 0,00256 & 0,00097 \\
\hline P7 & 30 & 0 & 0,02384 & 0,10674 & 0,00399 & 0,00144 \\
\hline P8 & 0 & -30 & 0,01987 & 0,04702 & 0,00240 & 0,00171 \\
\hline P9 & 0 & -20 & 0,01312 & 0,0119 & 0,00267 & 0,00121 \\
\hline P10 & 0 & -10 & 0,01006 & 0,02181 & 0,00209 & 0,00082 \\
\hline P11 & 0 & 10 & 0,00916 & 0,05223 & 0,00143 & 0,00045 \\
\hline P12 & 0 & 20 & 0,00930 & $\mathbf{0 , 0 1 0 1 5}$ & 0,00152 & $\mathbf{0 , 0 0 0 3 1}$ \\
\hline P13 & 0 & 30 & 0,00920 & 0,0132 & 0,00212 & 0,00048 \\
\hline
\end{tabular}


The figure 13 represents SAR with different head-antenna positions for 4 frequencies $900 \mathrm{MHz}$, $1800 \mathrm{MHz}, 2100 \mathrm{MHz}$ and $2600 \mathrm{MHz}$.

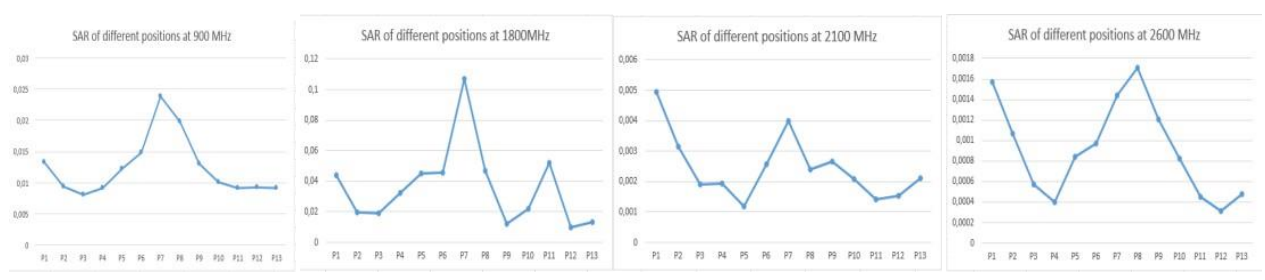

Fig. 13. SAR with different head-antenna positions for 4 frequencies $900 \mathrm{MHz}, 1800 \mathrm{MHz}, 2100 \mathrm{MHz}$ and $2600 \mathrm{MHz}$.

The table 4 represents optimum positions with $\theta$ xmax and $\theta y \max$ inclinations between the human head and the mobile phone for the four frequencies of $900 \mathrm{Mhz}, 1800 \mathrm{Mhz}, 2100 \mathrm{Mhz}$ and 2600Mhz.

Table 4. Optimum positions with $\theta_{x \max }$ and $\theta_{\mathrm{ymax}}$ inclinations between the human head and the mobile phone for the four frequencies.

\begin{tabular}{|c|c|c|}
\cline { 2 - 3 } \multicolumn{1}{c|}{} & \multicolumn{2}{c|}{ Optimum head-mobile position } \\
\hline Frequency & $\boldsymbol{\theta}_{\text {xmax }}$ & $\boldsymbol{\theta}_{\text {ymax }}$ \\
\hline $\mathbf{9 0 0 M H z}$ & $-10^{\circ}$ & $0^{\circ}$ \\
\hline $\mathbf{1 8 0 0 M H z}$ & $0^{\circ}$ & $20^{\circ}$ \\
\hline $\mathbf{2 1 0 0 M H z}$ & $10^{\circ}$ & $0^{\circ}$ \\
\hline $\mathbf{2 6 0 0 M H z}$ & $0^{\circ}$ & $20^{\circ}$ \\
\hline
\end{tabular}

\section{Conclusion}

The intensity of the SAR and the increase in temperature have important values if the antenna is also close to the human head and less important when it is a little far away. The intensity of the temperature rise has small values with little exposure time and significant for a long duration of exposure. The three layers: skin, muscle and CSF protect the brain as these tissues absorb most of the power emitted by the mobile phone. The study of the thermal effect of GSM on the human head and the human hand has given results for the SAR $(<1.42 \mathrm{~W} / \mathrm{Kg}$ and $<0.3 \mathrm{~W} / \mathrm{Kg})$ and for the temperature increase $\left(<1^{\circ} \mathrm{C}\right)$ are significantly lower compared to the maximum limits $(1.6 \mathrm{~W} / \mathrm{kg})$ [5] and $\left(4.5^{\circ} \mathrm{C}\right)[6]$ set by international standards. Our study confirmed the existence of optimal inclinations between the mobile phone and the human head. This confirms that our patch tetragenerations patch is compliant and meets the standards of dosimetry and temperature when used near the head and the human hand. 


\section{Acknowledgments}

We thank the members of the Institute of Electronics and Telecommunications Rennes (IETR) for their cooperation and help in the accomplishment of this work.

\section{References}

[1] J. Baayer, R. Karli, and H. Ammor : An Innovative Conception of a Compact Quadruple Frequency Microstrip Patch Antenna for Wireless and Mobile Communication. International Journal of Applied Engineering Research (IJAER). ISSN 0973-4562. Vol.10, $\mathrm{N}^{\circ} .5$ pp. 3979-3982(2015)

[2] P.J. Soh, G.A.E. Vandenbosch, F.H. Wee, A. Van Den Bosch, M. Martinez-Vazquez and D. Schreurs : Specific Absorption Rate (SAR) evaluation of biomedical telemetry textile antennas. Microwave Symposium Digest (IMS). IEEE MTT-S International. IEEE, pp. 1-3 (2013)

[3] Diao, Yinliang, Al, : Detailed modeling of palpebral fissure and its influence on SAR and temperature rise in human eye under $\mathrm{GHz}$ exposures. Bioelectromagnetics37.4 : 256263B(2016)

[4] R. Karli, H. Ammor and J. Terhzaz : Dosimetry in the human head for two types of mobile phone antennas at GSM frequencies. Central European Journal of Engineering.Vol. 4, N. 1. pp. 39-46 (2014)

[5] A. Hirata, K. Shirai and O. Fujiwara : An Averaging Mass of SAR Correlating with Temperature Elevation Due to a Dipole Antenna. Progress in Electromagnetics Research, PIER, 84, pp. 221-237 (2008)

[6] A. C. Guyton and J. E. Hall: Textbook of Medical Physiology. Philadelphia. PA: W. B. Saunders (1996) 
